\title{
Heterogeneity of tumor prognostic markers: a reproducibility study applied to liver metastases of pancreatic endocrine tumors
}

Anne Couvelard ${ }^{1}$, Lydia Deschamps ${ }^{1}$, Philippe Ravaud ${ }^{2}$, Gabriel Baron ${ }^{2}$, Alain Sauvanet ${ }^{3}$, Olivia Hentic ${ }^{4}$, Nathalie Colnot ${ }^{1}$, Valérie Paradis ${ }^{1}$, Jacques Belghiti ${ }^{3}$, Pierre Bedossa ${ }^{1}$ and Philippe Ruszniewski ${ }^{4}$

${ }^{1}$ Department of Pathology, University of Paris 7, Assistance Publique-Hôpitaux de Paris, Beaujon Hospital, Clichy, France; ${ }^{2}$ Department of Epidemiology, Biostatistics and Clinical Research, Bichat Hospital, Paris, France; ${ }^{3}$ Department of Surgery, University of Paris 7, Assistance Publique-Hôpitaux de Paris, Beaujon Hospital, Clichy, France and ${ }^{4}$ Department of Gastroenterology, University of Paris 7, Assistance PubliqueHôpitaux de Paris, Beaujon Hospital, Clichy, France

\begin{abstract}
Liver biopsy of metastatic pancreatic endocrine tumors allows confirmation of the diagnosis and assessment of prognosis. However, sampling variability is a potential limitation. Our aim was to use the tissue microarray technique to assess the heterogeneity of three prognostic markers, ie, MIB-1 proliferation index, microvascular density and somatostatin receptor type 2, inside single or between synchronous or metachronous liver metastases of pancreatic endocrine tumors. Tissue microarrays were constructed, which included core biopsies taken from surgically resected liver metastases in 29 patients. MIB-1, microvascular density and somatostatin receptor type 2 were evaluated after immunostaining. The heterogeneity was highlighted by the calculation of the reproducibility of the values of two cores randomly selected among all the cores studied. For quantitative variables, it was assessed by the intraclass correlation coefficient and by a Bland-Altman approach. For qualitative variables, observed agreement and weighted $\kappa$ were given. A total of 184 liver metastases were analyzed. For MIB-1, the intraclass correlation coefficients were $0.63,0.69$ and 0.67 and for microvascular density, the intraclass correlation coefficients were $0.48,0.60$ and 0.00 , respectively, in single, synchronous and metachronous liver metastases. The variability increased for higher mean values of microvascular density. For somatostatin receptor type 2, the observed agreements were $91 \%(\kappa=0.81), 69 \%$ $(\kappa=0.49)$ and $79 \%(\kappa=0.68)$ in single, synchronous and metachronous liver metastases, respectively. In conclusion, tissue microarray analysis identifies heterogeneity of protein expression in pancreatic endocrine metastases, which depends on the marker tested. The reproducibility is better for MIB-1 and somatostatin receptor type 2 than for microvascular density. Sampling variability should be taken into consideration as a potential limitation to the assessment of prognostic and therapeutic markers in biopsy samples from metastatic pancreatic endocrine tumors.
\end{abstract}

Modern Pathology (2009) 22, 273-281; doi:10.1038/modpathol.2008.177; published online 7 November 2008

Keywords: heterogeneity; reproducibility; tissue microarray; pancreatic endocrine tumors

Liver metastases have a strong prognostic impact in patients with pancreatic endocrine tumors. ${ }^{1-3}$ Liver biopsy is the main method available to assess diagnostic and prognostic markers and potential therapeutic targets. However, it is not known whether the assessment of prognostic factors in

Correspondence: Dr A Couvelard, Service d'Anatomie Pathologique, Hôpital Beaujon, 100 Boulevard du Général Leclerc, 92110 Clichy, France.

E-mail: anne.couvelard@bjn.aphp.fr

Received 10 July 2008; revised 6 October 2008; accepted 7 October 2008; published online 7 November 2008 small samples is representative of the entire metastases and of their biology. For example, the evaluation of the proliferative activity, as determined by MIB-l labeling index is important to predict the aggressiveness of primary or metastatic endocrine tumors. ${ }^{4-9}$ In some tumors, such as gastric stromal tumors, the heterogeneity in the expression of the proliferative index has been demonstrated. ${ }^{10}$ Sampling variability of MIB-1 labeling index, which could be a potential limitation to reliably predict outcome, has never been analyzed within endocrine metastases. Other markers have an impact in the prognosis or in the management of patients with 
pancreatic endocrine tumors, such as expression of somatostatin receptors or microvascular density. Immunohistochemical profiling of various somatostatin receptors subtypes as a part of routine pathologic analysis may become a useful predictor of responsiveness to various somatostatin analogs. ${ }^{11}$ Microvascular density is correlated with prognosis in many tumor types, including pancreatic endocrine tumors. ${ }^{12,13}$ Its quantification is heterogeneous in most cancer types and it is therefore mostly analyzed in hot spots, which correspond to areas with the highest vascular density. ${ }^{14-18}$ Moreover, microvascular density reflects tumor angiogenesis, which is a therapeutic target shared by many types of tumors.

Heterogeneity of protein expression among biopsy samples taken from the same tumor should be taken into account especially when molecular markers are used in decision making. Tissue microarray experiments allow large scales profiling of tissue samples. This technique allows multiple measurements of a given protein, similar to small biopsy samples, and then can be used to study biological heterogeneity of tumors. Our aim was to use this technique to assess the heterogeneity of MIB-l labeling index, microvascular density and somatostatin receptor type 2 expression in liver metastases of pancreatic endocrine tumors. Potential tumor heterogeneity was tested in three different conditions; ie, (a) within a single metastasis, (b) between synchronous metastases present at the same time in a patient and (c) between successive metastases in a patient.

\section{Materials and methods}

\section{Patients}

Hepatic surgical samples from 29 patients with metachronous or synchronous liver metastases from pancreatic endocrine tumors were studied. The patients were operated on in Beaujon Hospital between 1995 and 2006. All the metastases were histologically reviewed by two pathologists (AC and LD). All tumors were classified as well-differentiated endocrine carcinomas according to the World Health Organization (WHO) 2000 criteria. ${ }^{6,7}$ The following pathological characteristics were recorded for each metastasis: size, location in the liver, presence of necrosis and presence of cystic or hemorrhagic changes.

\section{Tissue Microarray Construction}

Four tissue microarrays were constructed from the liver metastases. Paraffin-embedded tissue blocks were selected for each metastasis. Tissue microarrays included several different 1-mm diameter core biopsies from each paraffin tissue block using a manual tissue-arraying instrument (Manual Tissue
Arrayer-MTA1; Beecher Instruments Inc.). The number of cores per nodules depended on the size of the latter: $1,2,3,4$ and 5 cores in nodules measuring $<0.5 \mathrm{~cm}, 0.5-2 \mathrm{~cm}, 2-5 \mathrm{~cm}, 5-8 \mathrm{~cm}$ and $>8 \mathrm{~cm}$, respectively. The cores were taken at random inside the nodules. In total 418 cores were included in the 4 tissue blocks.

\section{Staining Methods}

Antibodies and Immunohistochemical Techniques The three antibodies are reactive in paraffinembedded sections. Ki-67 protein was detected with murine monoclonal antibody MIB-1 (MIB-1; Dako, Glostrup, Denmark). The quantification of microvessel density was performed after immunostaining with CD34 antibody (QBEND10; Immunotech, Marseille, France). The somatostatin receptor type 2A (SSTR2A) was detected with rabbit polyclonal antibody (Biotrend, Köln, Germany). An automatized technique (Streptavidine-peroxidase with an automate Ventana Benchmark, USA) was used for the three antibodies. Antigen retrieval was conducted by pretreatment with high temperature.

\section{Scoring Methods}

The proliferation index was calculated after immuno staining with MIB-1 antibody. The results were presented as the percentage of nuclear stained tumor cells in each core. Microvascular density evaluation was performed after immunostaining with CD34 antibody. Vessels with a clearly defined lumen or well-defined linear vessel shape were taken into account for counting. The vessels were counted in each $1 \mathrm{~mm}$ diameter core and the results were reported for $1 \mathrm{~mm}^{2}$. The results were also categorized in three groups: $\leq 200,200-400$ and $>400$ microvessels per $\mathrm{mm}^{2}$. Immunohistochemical staining of somatostatin receptor type 2 was evaluated independently by two pathologists. The intensity of staining was noted (negative scored as 0 ; weak or moderate, which corresponded to the intensity of the staining of the normal islets, scored as 1; strong scored as 2). Discrepancies were resolved by simultaneous reexamination of the slides by both investigators using a double-headed microscope.

\section{Study of Reproductibility}

\section{Heterogeneity assessment}

Three types of heterogeneity were tested: (a) inside single metastases (intrametastasis heterogeneity) in metastases $\geq 2 \mathrm{~cm}$ only, (b) between metastases resected at the same time in the same patient (synchronous intermetastases heterogeneity) and (c) between metastases present at two different periods in patients undergoing iterative hepatectomy (metachronous intermetastases heterogeneity). To assess the heterogeneity two cores 
were randomly selected among all the cores available (two cores from the same metastasis for intrametastasis heterogeneity, two cores from separate metastases resected at the same time for synchronous intermetastases heterogeneity, two cores from separate metastases present at two successive time for metachronous intermetastases heterogeneity).

\section{Intraobserver and interobserver variability}

One core taken at random in each patient was selected to perform intraobserver and interobserver variability. The semiquantitative assessment of somatostatin receptor type 2 intensity (graded into three classes) based on the pathologist's judgment and therefore in part subjective was analyzed by two observers. Intraobserver and interobserver variability were calculated for this marker. One observer calculated the Ki-67 index and the number of microvessels. Intraobserver variability was calculated for these two markers. The second analysis for intraobserver variability was performed 15 days after the first one.

\section{Statistical Analysis}

To assess variability in case of quantitative variables (for microvascular density and index of proliferation evaluation), the intraclass correlation coefficient was given. Intraclass correlation coefficient values range from 0 (totally unreproducible) to 1 (perfectly reproducible). ${ }^{19}$ A 95\% confidence interval (95\% CI) was estimated by a bootstrap method. Variability between quantitative variables was also assessed by a Bland-Altman approach. ${ }^{20}$ The difference between the two randomly selected measures was plotted against the average of the two randomly selected measures, and limits of agreement involving the mean difference and the s.d. of the difference were computed and plotted. By definition, if differences are normally distributed, 95\% of individual differences are within 2 s.d. of the mean difference (ie, within the limits of agreement). The Bland-Altman plot is useful to search for any systematic bias, assess random error and reveal whether the difference between scores depends on the level of scores.

In case of qualitative variables (for somatostatin receptor type 2 and microvascular density evaluation), observed agreement and weighted $\kappa$ were given, with a $95 \%$ CI. The strength of agreement computed with weighted $\kappa$ was labeled as follows: value $\leq 0.40$, poor to fair agreement; 0.41 through 0.60 , moderate agreement; 0.61 through 0.80 , substantial agreement; 0.81 through 1.00, almost perfect

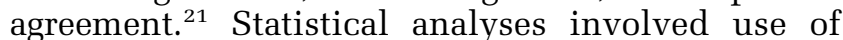
SAS software, version 9.1 (SAS Institute, Cary, NC, USA) and R, version 2.2.0.

\section{Results}

\section{Patients' Characteristics}

A total of 29 patients were included. Five patients presented with a functioning syndrome (insulinoma, $n=1$; glucagonoma, $n=2$; gastrinoma, $n=1$; somatostatinoma, $n=1$ ) and one patient had multiple endocrine neoplasia type 1 disease. In 17 patients, liver metastases were resected during pancreatectomy. In 23 patients, metastases were resected after the pancreatectomy. Three resections corresponded to liver transplantations. Of the patients, 15 had only one hepatic surgical resection (either during the pancreatectomy or after), 14 had at least two successive hepatic resections. In total, 184 metastases were analyzed (size, 0.5-12 cm; median: $1.5 \mathrm{~cm})$. The number of synchronous metastases varied from 1 to 12 (median: 4). The number of metastases in a single patient varied from 1 to 19 .

\section{Localization and Aspects of the Metastases}

Of the metastases, 55 were located in the left lobe (segments II and III), 26 in segment IV, 7 in segment I and 96 in the right liver (segments V, VI, VII and VIII); 39 metastases contained a fibrous stroma, 9 were cystic and 17 hemorrhagic. All metastases corresponded to well-differentiated endocrine carcinomas, according to the WHO classification.

\section{Immunohistochemical Analysis}

Of 418 cores, 384 were analyzed for MIB-1 expression. (Main results are given in Table 1.) The counting was not feasible in 34 cores because of the absence of tumor tissue in the cores or of tissue drop during the technique. The proliferation index varied from 0 to $36.8 \%$. The median values for each patient varied from 0.05 to $19.9 \%$ (Figure $1 \mathrm{a}$ and $\mathrm{b}$ ).

Of 428 cores, 379 were analyzed for CD34 expression. The counting of CD34 + vessels was not feasible in 49 cores because of the absence of tumor tissue in the cores or of tissue drop during the technique. The microvascular density varied from 5 to 1620 vessels per $\mathrm{mm}^{2}$. The median values for each patient varied from 56 to 755 per $\mathrm{mm}^{2}$. Of 379 cores, 182 were classified as 1, 142 as 2 and 55 as 3 (Figure 1c and d).

Of 418 cores, 406 were analyzed for somatostatin receptor type 2 expression. The counting was not feasible in 12 cores because of the absence of tumor tissue in the cores or of tissue drop during the technique. In all cores, the positive staining was detected on $100 \%$ of the epithelial cells. The intensity was classified as follows in the 406 cores: 57 as 0 (no staining), 193 as 1 and 156 as 2 (Figure 1e and f).

\section{Heterogeneity Assessment}

Intrametastasis heterogeneity was tested in 24 patients with at least one metastasis $\geq 2 \mathrm{~cm}$. Because 
Table 1 Median, minimum and maximum values of MIB-1, microvascular density and somatostatin receptors type 2 for each patient

\begin{tabular}{|c|c|c|c|c|c|c|c|c|c|c|}
\hline \multirow[t]{2}{*}{ Patient } & \multirow[t]{2}{*}{ Nmet } & \multirow[t]{2}{*}{ Ncore } & \multicolumn{3}{|c|}{$M I B-1$} & \multicolumn{3}{|c|}{ Microvascular density (CD34) } & \multicolumn{2}{|c|}{ Somatostatin receptor type 2} \\
\hline & & & Min. & Med. & Max. & Min. & Med. & $\operatorname{Max}$ & Min. & Max. \\
\hline P1 & 7 & 17 & 0.22 & 2.13 & 3.07 & 125 & 160 & 359 & 2 & 2 \\
\hline P2 & 1 & 4 & 5.56 & 6.46 & 8.44 & 42 & 76 & 230 & 2 & 2 \\
\hline P3 & 5 & 16 & 0 & 0.53 & 11.5 & 6 & 56 & 1620 & 0 & 1 \\
\hline P4 & 8 & 17 & 0 & 2.49 & 12.5 & 7 & 122 & 392 & 1 & 2 \\
\hline P5 & 6 & 12 & 0 & 0.59 & 2.5 & 40 & 206 & 603 & 0 & 2 \\
\hline P6 & 2 & 4 & 0.75 & 1.25 & 2.85 & 356 & 755 & 1120 & 1 & 1 \\
\hline P7 & 7 & 14 & 0 & 0.05 & 1.7 & 41 & 86 & 428 & 0 & 2 \\
\hline P8 & 3 & 4 & 1.40 & 2.33 & 4.5 & 198 & 272 & 320 & 2 & 2 \\
\hline P9 & 12 & 23 & 0.28 & 4.47 & 16 & 220 & 453 & 790 & 0 & 1 \\
\hline P10 & 9 & 19 & 0 & 0.22 & 0.83 & 216 & 575 & 1120 & 1 & 2 \\
\hline P11 & 8 & 15 & 2.38 & 4.31 & 6.39 & 36 & 81 & 288 & 0 & 0 \\
\hline P12 & 5 & 15 & 0 & 5.06 & 10 & 91 & 220 & 990 & 2 & 2 \\
\hline P13 & 4 & 8 & 1.65 & 1.83 & 2 & 76 & 134 & 205 & 2 & 2 \\
\hline P14 & 1 & 2 & 2.14 & 2.21 & 2.29 & 112 & 129.5 & 147 & 1 & 2 \\
\hline P15 & 9 & 23 & 0.13 & 3.1 & 23.08 & 14 & 117 & 346 & 1 & 2 \\
\hline P16 & 7 & 16 & 3 & 4.64 & 10 & 110 & 203.5 & 324 & 1 & 1 \\
\hline P17 & 16 & 32 & 0 & 0.25 & 1.33 & 144 & 282.5 & 630 & 0 & 2 \\
\hline P18 & 8 & 17 & 1 & 5.15 & 13.30 & 13 & 101 & 132 & 1 & 2 \\
\hline P19 & 5 & 9 & 13.83 & 19.92 & 27.18 & 100 & 210 & 605 & 1 & 1 \\
\hline $\mathrm{P} 20$ & 9 & 19 & 0 & 2 & 36.8 & 84 & 216 & 450 & 1 & 2 \\
\hline P21 & 2 & 4 & 0.5 & 0.58 & 0.79 & 345 & 472.5 & 72 & 1 & 1 \\
\hline $\mathrm{P} 22$ & 19 & 35 & 0.30 & 5 & 12 & 82 & 168 & 775 & 1 & 2 \\
\hline P23 & 9 & 17 & 0 & 0.33 & 0.75 & 215 & 485 & 700 & 2 & 2 \\
\hline P24 & 3 & 3 & 7.5 & 8.1 & 9. & 126 & 151 & 180 & 0 & 0 \\
\hline $\mathrm{P} 25$ & 4 & 10 & 1 & 2.88 & 6.7 & 90 & 270 & 430 & 2 & 2 \\
\hline P26 & 6 & 8 & 0 & 0.28 & 0.45 & 295 & 515 & 1100 & 1 & 1 \\
\hline P27 & 5 & 17 & 0 & 1.4 & 10.5 & 5 & 70 & 360 & 1 & 2 \\
\hline P28 & 9 & 22 & 0.2 & 0.8 & 25 & 36 & 412 & 1342 & 1 & 1 \\
\hline P29 & 3 & 5 & 1.11 & 2.91 & 3.11 & 265 & 310 & 590 & 0 & 2 \\
\hline
\end{tabular}

Nmet: number of metastases; Ncore: number of microarray cores tested; Min.: minimum; Med.: median; Max.: maximum.

Microvascular density is measured in vessels $/ \mathrm{mm}^{2}$.

of the absence of tumor tissue in the cores or of tissue drop during the technique in some cases, 39, 37 and 42 metastasis were analyzed for MIB-1, CD34 and somatostatin receptor type 2, respectively. Synchronous intermetastases heterogeneity was analyzed in 27 patients presenting at least two metastases resected at the same time. It was tested 35, 36 and 36 times for MIB-1, CD34 and somatostatin receptor type 2, respectively. Metachronous intermetastases heterogeneity was analyzed in the 14 patients who had at least two successive surgical resections.

MIB-1. The intraclass correlation coefficient was $0.63(95 \% \mathrm{CI}=(0.09 ; 0.83)), 0.69(95 \% \mathrm{CI}=(0.27$; $0.91)$ ), and 0.67 (95 $\mathrm{CI}=(0.00 ; 0.91))$, in single, synchronous or metachronous metastases respectively (Figure 2a-c). The analysis of the Bland-Altman plots (Figure 2) showed that, in metachronous metastases, in each three types of heterogeneity tested, the mean differences were close to zero indicating that there was no bias. It revealed that the range between the two limits of agreements were similar in the three groups tested indicating the same variability in these three conditions. The Bland-Altman plots show that the variability increased for higher mean values of MIB-1.
Microvascular density

Qualitative Data. The observed agreement was of $81 \%(\kappa=0.66), 67 \%(\kappa=0.54)$ and $57 \%(\kappa=0.23)$ in single, synchronous and metachronous metastases, respectively.

Quantitative Data. The intraclass correlation coefficient was of $0.48(95 \% \mathrm{CI}=(0.00 ; 0.78)), 0.60(95 \%$ $\mathrm{CI}=(0.40 ; 0.79))$ and $0.00(95 \% \mathrm{CI}=(0.00 ; 0.30))$ in single, synchronous and metachronous metastases, respectively (Figure 2d-f). The analysis of the BlandAltman plots (Figure 3) showed that, in each three types of heterogeneity tested, the mean differences were close to zero indicating that there was no bias. It revealed that the range between the two limits of agreements was higher in the metachronous metastases group, indicating a higher variability in this condition. The BlandAltman plots also show that the variability increased for higher mean values of microvascular density.

Somatostatin Receptor Type 2. The observed agreement was of $91 \%(\kappa=0.81), 69 \% \quad(\kappa=0.49)$ and $79 \% \quad(\kappa=0.68)$ in single, synchronous and metachronous metastasis, respectively.

\section{Intraobserver and Interobserver Variability}

A total of 29 cores (one per patient) were selected to perform this analysis. 

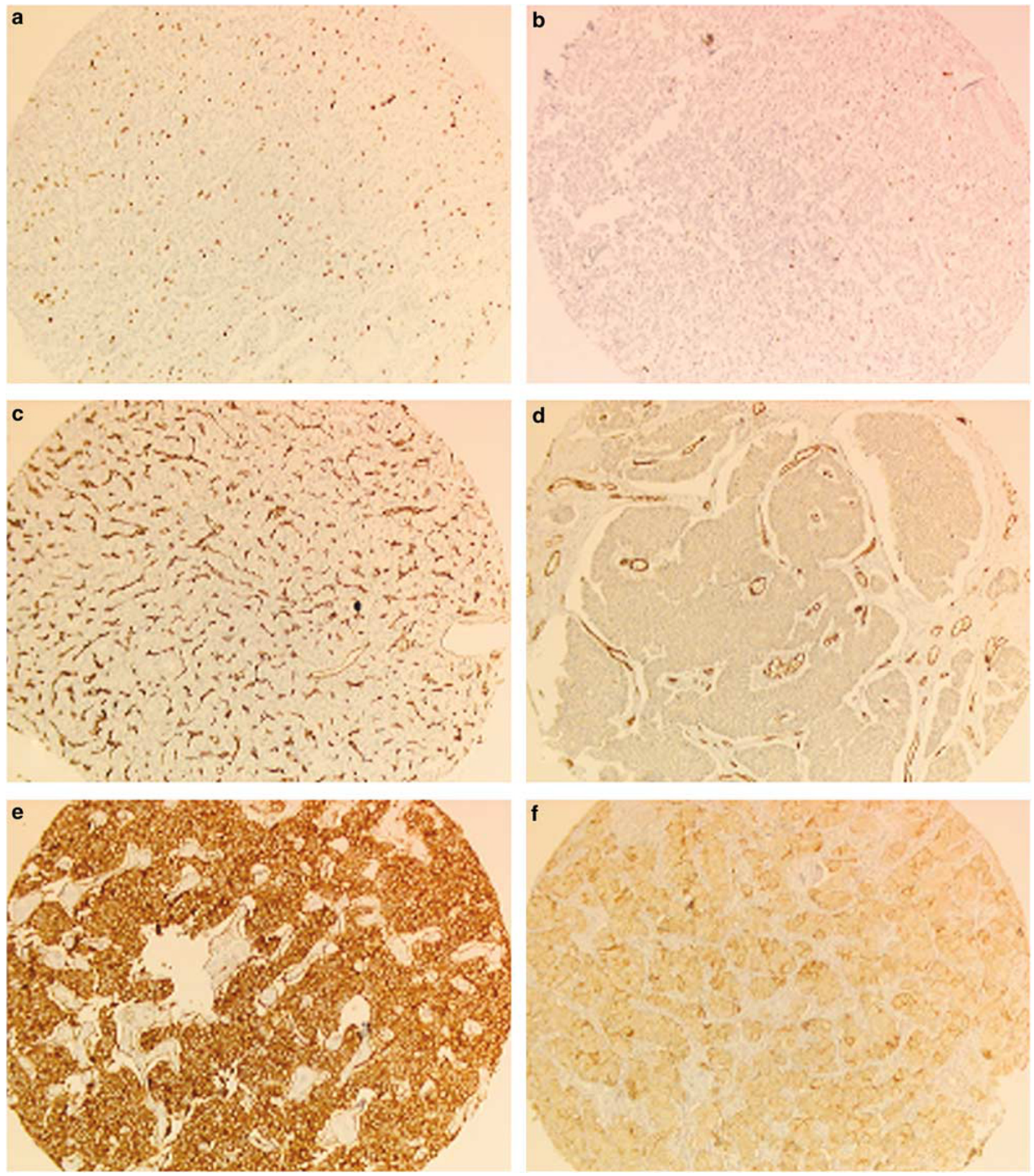

Figure 1 Immunohistochemical expression of MIB-1 (a, b), CD34 (c, d) and somatostatin receptor type 2 (e, f) by liver metastases of pancreatic endocrine tumors. Panels, $\mathbf{a}, \mathbf{c}, \mathbf{e}$ and $\mathbf{b}, \mathbf{d}, \mathbf{f}$ show high and low expression of the three markers, respectively. (a, b) MIB-1 nuclear expression is high, calculated at $12 \%$ in panel (a) and is low, calculated at $2 \%$ in panel (b). (c, d) CD34 + capillaries are numerous (microvascular density $=880$ vessels per $\mathrm{mm}^{2}$ ) in panel $(\mathbf{c})$ and rare (microvascular density $=129$ vessels per mm ${ }^{2}$ ) in panel (d). (e, f) Somatostatin receptor type 2 is strongly (intensity 2) expressed in panel (e) and faintly expressed (intensity 1) in panel (f). (a-e) Immunoperoxidase and hematoxylin nuclear counterstaining.

$M I B-1$

The intraobserver intraclass correlation coefficient for MIB-1 index was $0.99(95 \% \mathrm{CI}=(0.96 ; 1.00))$.

\section{Microvascular density}

The intraobserver intraclass correlation coefficient for microvascular density as a continuous variable 


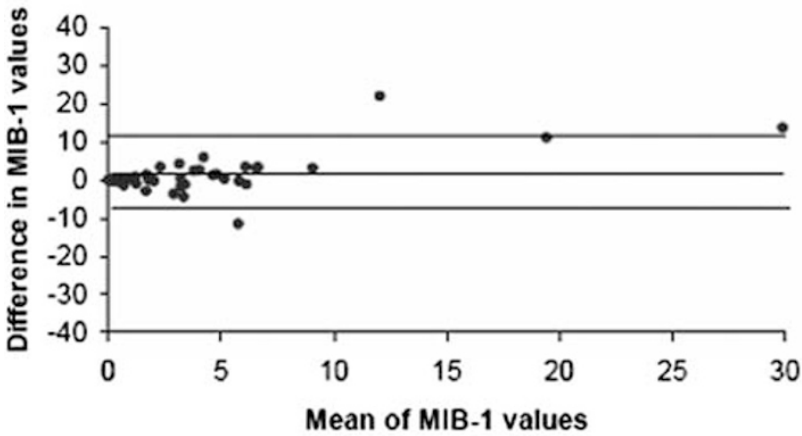

b Synchronous inter-metastases heterogeneity

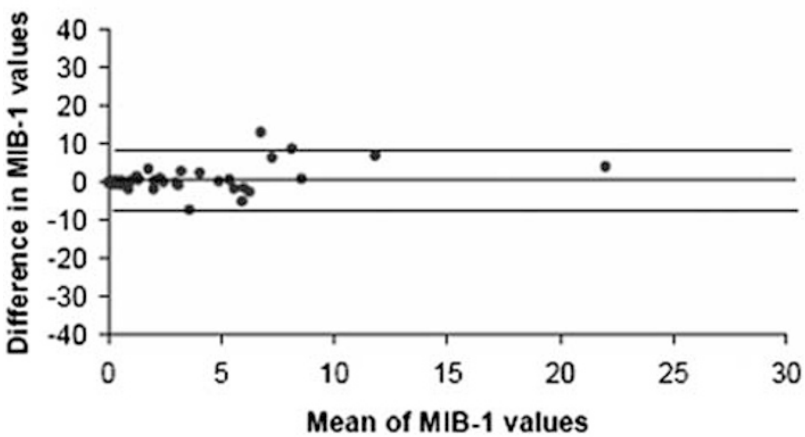

c Metachronous inter-metastases heterogeneity

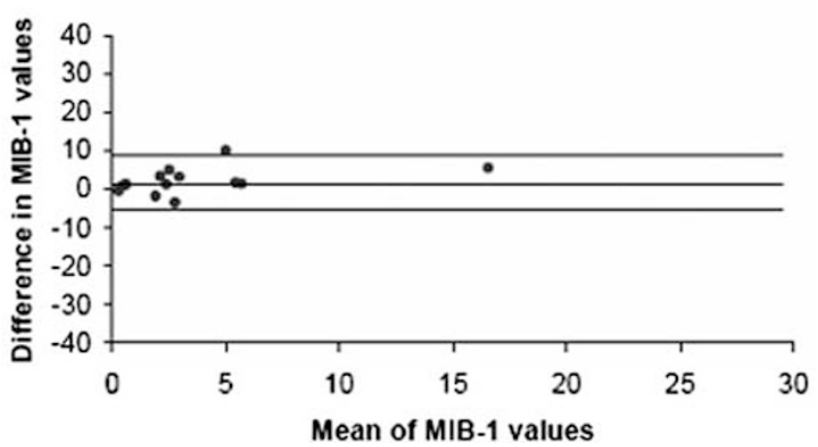

Figure 2 Bland-Altman plot of difference between the two randomly selected measures by mean of the two randomly selected measures for MIB-1 in the three types of heterogeneity tested: (a) intrametastasis heterogeneity; (b) synchronous intermetastases heterogeneity; (c) metachronous intermetastases heterogeneity. The three horizontal lines stand for mean individual difference \pm 2 s.d. The limits of agreement were of $(-8,81 ; 11,62)$, $(-6,3 ; 8,1)$ and $(-4,5 ; 8,5)$, respectively, for the three types of heterogeneity tested.

was $0.98(95 \% \mathrm{CI}=(0.94 ; 0.99))$. The intraobserver observed concordance for microvascular density as a qualitative variable was $90-95 \% \quad \mathrm{CI}=(73 ; 98)$ $(\kappa=0.87 ; 95 \% \mathrm{CI}=(0.74 ; 1.00))$.

\section{Somatostatin receptor type 2}

The intraobserver concordance was 97\%, 95\% $\mathrm{CI}=(82 ; 100)(\kappa=0.96 ; 95 \% \mathrm{CI}=(0.88 ; 1.00))$ and a

Intra-metastasis heterogeneity

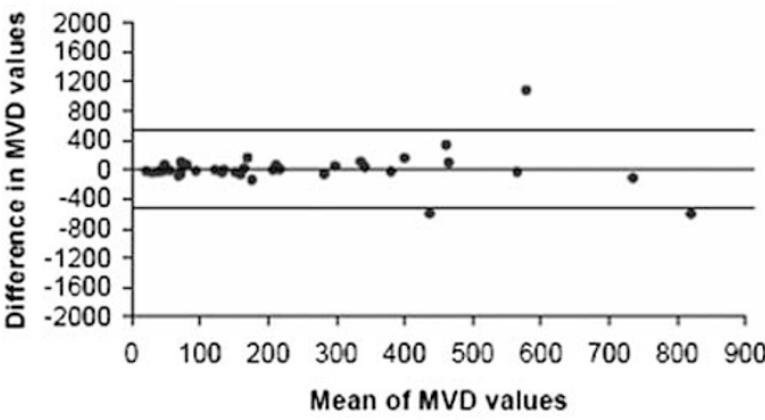

b Synchronous inter-metastases heterogeneity

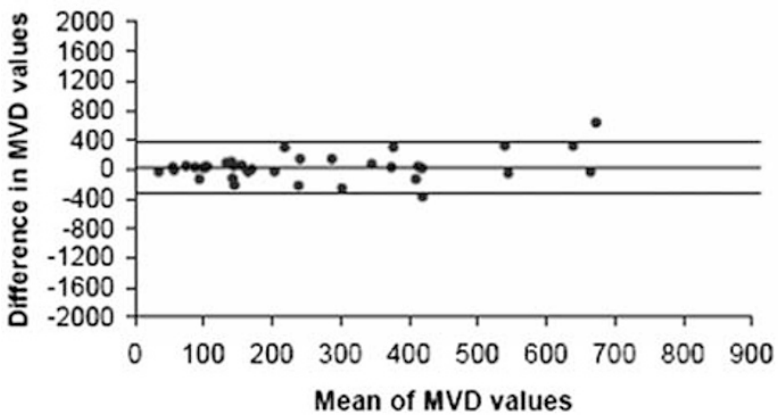

C Metachronous inter-metastases heterogeneity

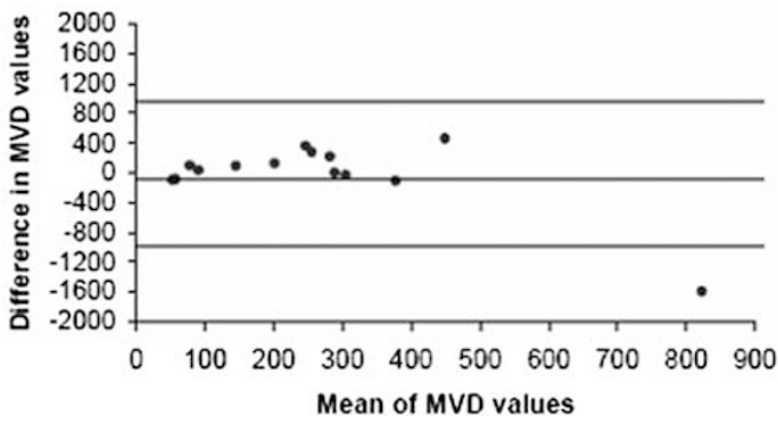

Figure 3 Bland-Altman plot of difference between the two randomly selected measures by mean of the two randoml selected measures for microvascular density in the three types of heterogeneity tested: (a) intrametastasis heterogeneity; (b) synchronous intermetastases heterogeneity; (c) metachronous intermetastases heterogeneity). The three horizontal lines stand for mean individual difference \pm 2 s.d. The limits of agreement were of $(-470 ; 590),(325 ; 398)$ and $(-970 ; 938)$, respectively, for the three types of heterogeneity tested.

the interobserver concordance was 83\%, 95\% $\mathrm{CI}=(64 ; 94)(\kappa=0.79 ; 95 \% \mathrm{CI}=(0.61 ; 0.96))$.

\section{Discussion}

In this study we used the tissue microarray technique to evaluate the heterogeneity of expression of a panel of proteins that play a role in tumor 
progression and have the potential to be translatable to a clinical application, in patients with liver metastases of pancreatic endocrine tumors. The aim was to determine to what extent the performance of liver biopsy could be influenced by sampling limitation. To achieve this goal, we compared the percentage of agreement between values of a couple of cores selected at random in each metastasis or in several metastases in the same patient. The observed discrepancies were attributed to tumor heterogeneity.

In most cases, the tissue microarray technique has been used to allow the examination of large tissue cohorts to spare material and technical resources and with increased reproducibility. Many reports have pointed out that the material in the tissue cores are not always fully representative of the diagnostic section and found discrepant core results, attributed to tumor heterogeneity. ${ }^{22,23}$ The agreement improves by using an average measurement of replicate samples. For example, this has been demonstrated for Ki-67 expression in different tumor types. ${ }^{24,25}$ However, an average measurement hides the heterogeneity present in replicates, ignoring variation among biopsy samples. Here, we used the tissue microarray technique to assess the heterogeneity inside liver metastases of pancreatic endocrine tumors. This variability can be attributable to both sampling and observer. In this study, we demonstrated a low degree of intra- or interobserver variability, which allows attributing the variability to the sampling.

Evaluation of the MIB-1 labeling index by immunohistochemistry on paraffin sections, is the gold standard for assessment of endocrine tumor progression. ${ }^{5-9}$ Liver biopsies allow estimating this index in metastatic endocrine tumors and the therapeutic decision depends mainly on this criterion. For example, it is regarded as a valuable tool to adapt systemic chemotherapy or to identify patients who will profit from liver transplantation. ${ }^{26}$ However, data are lacking concerning the heterogeneity of the expression of MIB-1 either in primary or metastatic pancreatic endocrine tumors. Although the prognosis of the disease is mainly due to the progression of liver metastases, it is not known whether the MIB-1 labeling index is variable among a given metastasis or between several metastases in the same patient and if it varies with time progression. Our results show an intraclass correlation coefficient of 0.63 , 0.69 and 0.67 if heterogeneity was tested inside single metastasis, synchronous and metachronous metastases, respectively, indicating a substantial reproducibility for this marker. Interestingly, the reproducibility is not much modified when several synchronous metastases are taken into account in the same patient, as compared to the reproducibility inside single metastases, as shown by the homogeneous limits of agreement in the Bland-Altman approach. This suggests that there is no more variation in the index of proliferation between synchronous metastases than inside single metastasis. According to this result, the selection of the metastasis to biopsy among all metastases present at a given time in a patient should be only based on its accessibility. Moreover, to limit the risk for false evaluation of MIB-1 labeling index, it is probably as much effective to increase the number of passes performed within a liver metastasis than to biopsy several metastases. In the same way, we also show that the reproducibility is not modified for successive metastases as compared to intrametastasis heterogeneity, suggesting that there is no important modification of the proliferation index with time in a patient. However it must be kept in mind that the liver metastases were all resected, probably excluding the highly proliferating ones. Interestingly, a consensus report recently suggested to perform the evaluation of the MIB-1 index in 2000 cells in digestive endocrine tumors. ${ }^{9}$ Counting the percentage of positive cells in a 1-mm core seems relevant because such area approximately contains this number of tumor cells and the tissue core could be extrapolated to tumor tissue in a small liver biopsy. However, the authors recommend counting the index of proliferation in hot spots, ie, areas with the highest staining, which is possible only if representative tissue is analyzable.

The microvascular density evaluated by immunohistochemistry with the CD34 antibody is correlated with prognosis and survival in pancreatic endocrine tumors $^{4,18}$ Interestingly, it is correlated with contrast enhancement on CT scan which offers the possibility to assess endocrine tumor aggressiveness at the time of diagnosis. ${ }^{27}$ Our study shows a moderate intraclass correlation coefficient of 0.48 inside single metastasis, demonstrating that there is a substantial variability in the microvascular density of endocrine metastases when it is evaluated on small, $1 \mathrm{~mm}$ diameter areas. This is in accordance with the fact that the values of microvascular density vary greatly according to the area in most tumor types and the homogeneous range between the two limits of agreement. ${ }^{28-30}$ The analysis of the Bland-Altman plots also demonstrated that in each of the three types of heterogeneity tested, the variability increases for higher mean values of microvessels. Tumor vasculature is not well organized in tumors as compared to normal tissues and some areas are much less vascularized and hypoxic than others. ${ }^{31}$ All the above results are in accordance with many previous reports in several tumor types showing that the counting of the vessels is mostly reliable and correlated to prognosis if it is not performed randomly but evaluated in areas of highest vascular density. ${ }^{14-18}$ The unreproducible results obtained for metachronous metastases suggest that the vascularization is modified with time progression, possibly as a result of the systemic treatment, as observed in other carcinomas. ${ }^{32}$ Finally, we observed better agreements with the microvascular density as a qualitative data which 
probably reflects that using three groups (low, moderate or high counting) could help to separate highly from low vascularized tumors in good correlation with imaging contrast enhancement, prognosis and, hopefully, response to antiangiogenic treatments. ${ }^{27}$

Endocrine tumors usually express somatostatin receptors, although the subtypes and number of somatostatin receptors expressed in a certain tumor is very variable. However, somatostatin receptor type 2 is the major somatostatin receptor subtype in pancreatic endocrine tumors. ${ }^{33-35}$ The expression of somatostatin receptors and their density of expression on tumor cells form the basis for uptake of cold or radiolabeled somatostatin analogues and offer the possibility to diagnose or treat malignant pancreatic endocrine tumors, especially those with unresectable liver metastases. ${ }^{36,37}$ The investigation of the quantitative expression of somatostatin receptors allows evaluating the possibilities for tumor-specific radionucleotide therapy. Patients considered for radiolabeled somatostatin analog treatment should undergo liver biopsy to establish the immunohistochemical somatostatin receptors status of the liver metastases to treat. By this approach, it could be possible to select patients more suitable for treatment. In our study, we demonstrate that the expression of somatostatin receptors type 2 is homogeneous and reproducible in pancreatic endocrine tumor metastases. The overall concordance of the values tested was $90.5 \%$ among single metastasis, with a $\kappa$-value of 0.81 , indicating a very good agreement or a low heterogeneity. This suggests that the quantification of SSTR2 in a small biopsy sample can assign the correct expression level of a liver metastasis and could help to predict its responsiveness to various somatostatin receptor analogs. The lower agreement observed for synchronous intermetastases may explain that certain liver metastases do not respond as well as others to somatostatin analog treatment in some patients.

In conclusion, sampling errors of prognostic markers evaluation should be taken into consideration in therapeutic trial in patients with liver metastases of pancreatic endocrine tumors. The importance of the heterogeneity depends on the marker tested. Interestingly, our results point out that the reproducibility of MIB-1 labeling index and somatostatin receptor type 2 is better than the one of microvascular density. These results are important because these markers can be evaluated in biopsy samples to adapt therapeutic strategy in metastatic pancreatic endocrine tumors.

\section{Acknowledgement}

This work was supported by a grant from the 'Fonds de recherche de la Société Nationale Française de Gastroentérologie, SNFGE'.

\section{Disclosure/conflicts of interest}

No conflicts of interest exist.

\section{References}

1 Madeira I, Terris B, Voss M, et al. Prognostic factors in patients with endocrine tumours of the duodenopancreatic area. Gut 1998;43:422-427.

2 Plockinger U, Rindi G, Arnold R, et al. Guidelines for the diagnosis and treatment of neuroendocrine gastrointestinal tumours. A consensus statement on behalf of the European Neuroendocrine Tumour Society (ENETS). Neuroendocrinology 2004;80: 394-424.

3 Tomassetti P, Campana D, Piscitelli L, et al. Endocrine pancreatic tumors: factors correlated with survival. Ann Oncol 2005;16:1806-1810.

4 Clarke MR, Baker EE, Weyant RJ, et al. Proliferative Activity in Pancreatic Endocrine Tumors: Association with Function, Metastases, and Survival. Endocr Pathol 1997;8:181-187.

5 Gentil Perret A, Mosnier JF, Buono JP, et al. The relationship between MIB-1 proliferation index and outcome in pancreatic neuroendocrine tumors. Am J Clin Pathol 1998;109:286-293.

6 Solcia E, Klöppel G, Sobin LH, (eds). Histological Typing of Endocrine Tumours, 2nd edition. WHO International Histological Classification of Tumours. Springer: Berlin, 2000.

7 DeLellis RA, Lloyd RV, Heitz PU, et al. (eds). World Health Organisation Classification of Tumours: Pathology and Genetics of Tumours of Endocrine Organs. IARC Press: Lyon, 2004.

8 Rindi G, Couvelard A, Scoazec JY, et al. Practical notes for the evaluation of malignancy in digestive endocrine tumors. Ann Pathol 2005;25:487-498.

9 Rindi G, Kloppel G, Alhman H, et al., all other Frascati Consensus Conference participants; European Neuroendocrine Tumor Society (ENETS). TNM staging of foregut (neuro)endocrine tumors: a consensus proposal including a grading system. Virchows Arch 2006;449:395-401.

10 Yasui $\mathrm{H}$, Konishi E, Urasaki $\mathrm{K}$, et al. Intratumoral heterogeneity of MIB-1 labelling index in gastric gastrointestinal stromal tumor (GIST). Gastric Cancer 2006;9:223-228.

11 Nasir A, Stridsberg M, Strosberg J, et al. Somatostatin receptor profiling in hepatic metastases from small intestinal and pancreatic neuroendocrine neoplasms: immunohistochemical approach with potential clinical utility. Cancer Control 2006;13:52-60.

12 Marion-Audibert AM, Barel C, Gouysse G, et al. Low microvessel density is an unfavorable histoprognostic factor in pancreatic endocrine tumors. Gastroenterology 2003;125:1094-1104.

13 Couvelard A, O'Toole D, Turley H, et al. Microvascular density and hypoxia-inducible factor pathway in pancreatic endocrine tumours: negative correlation of microvascular density and VEGF expression with tumour progression. Br J Cancer 2005;92:94-101.

14 Maeda K, Chung YS, Takatsuka S, et al. Tumour angiogenesis and tumour cell proliferation as prognostic indicators in gastric carcinoma. Br J Cancer 1995;72:319-323. 
15 De Jong JS, van Diest PJ, Baak JP. Heterogeneity and reproducibility of microvessel counts in breast cancer. Lab Invest 1995;73:922-926.

16 De Jong JS, van Diest PJ, Baak JP. Hot spot microvessel density and the mitotic activity index are strong additional prognostic indicators in invasive breast cancer. Histopathology 2000;36:306-312.

17 Prall F, Gringmuth U, Nizze $\mathrm{H}$, et al. Microvessel densities and microvascular architecture in colorectal carcinomas and their liver metastases: significant correlation of high microvessel densities with better survival. Histopathology 2003;42:482-491.

18 Offersen BV, Borre M, Overgaard J. Quantification of angiogenesis as a prognostic marker in human carcinomas: a critical evaluation of histopathological methods for estimation of vascular density. Eur J Cancer 2003;39:881-890.

19 Shrout PE, Fleiss JL. Intraclass correlations: uses in assessing rater reliability. Psychol Bull 1979;86: 420-428.

20 Bland JM, Altman DG. Statistical methods for assessing agreement between two methods of clinical measurement. Lancet 1986;1:307-310.

21 Landis JR, Koch GG. The measurement of observer agreement for categorical data. Biometrics 1977;33: 159-174.

22 Gillett CE, Springall RJ, Barnes DM, et al. Multiple tissue core arrays in histopathology research: a validation study. J Pathol 2000;192:549-553.

23 Milanes-Yearsley M, Hammond ME, Pajak TF, et al. Tissue micro-array: a cost and time-effective method for correlative studies by regional and national cancer study groups. Mod Pathol 2002;15:1366-1373.

24 Hoos A, Urist MJ, Stojadinovic A, et al. Validation of tissue microarrays for immunohistochemical profiling of cancer specimens using the example of human fibroblastic tumors. Am J Pathol 2001;158:1245-1251.

25 Nocito A, Bubendorf L, Tinner EM, et al. Microarrays of bladder cancer tissue are highly representative of proliferation index and histological grade. J Pathol 2001;194:349-357.

26 Rosenau J, Bahr M, von Wasielewski R, et al. Ki-67, E-cadherin, and p53 as prognostic indicators of long- term outcome after liver transplantation for metastatic neuroendocrine tumors. Transplantation 2002;73: 386-394.

27 Rodallec M, Vilgrain V, Couvelard A, et al. Endocrine pancreatic tumours and helical CT: contrast enhancement is correlated with microvascular density, histoprognostic factors and survival. Pancreatology 2006; 6:77-85.

28 Fox SB. Tumour angiogenesis and prognosis. Histopathology 1997;30:294-301.

29 Fox SB, Harris AL. Histological quantitation of tumour angiogenesis. APMIS 2004;112:413-430.

30 Trivella M, Pezzella F, Pastorino U, et al. Prognosis In Lung Cancer (PILC) Collaborative Study Group. Microvessel density as a prognostic factor in non-small-cell lung carcinoma: a meta-analysis of individual patient data. Lancet Oncol 2007;8:488-499.

31 McDonald DM, Choyke PL. Imaging of angiogenesis. Nat Med 2003;9:713-725.

32 Bottini A, Berruti A, Bersiga A, et al. Changes in microvessel density as assessed by CD34 antibodies after primary chemotherapy in human breast cancer Clin Cancer Res 2002;8:1816-1821.

33 Reubi JC, Kappeler A, Waser B, et al. Immunohistochemical localization of somatostatin receptor sst2A in human pancreatic islets. J Clin Endocrinol Metab 1998;83:3746-3749.

34 Kimura N, Pilichowska M, Date F, et al. Immunohistochemical expression of somatostatin type 2A receptor in neuroendocrine tumors. Clin Cancer Res 1999; $5: 3483-3487$

35 Oda Y, Tanaka Y, Naruse T, et al. Expression of somatostatin receptor and effects of somatostatin analog on pancreatic endocrine tumors. Surg Today 2002;32:690-694.

36 Kubota A, Yamada Y, Kagimoto S, et al. Identification of somatostatin receptor subtypes and an implication for the efficacy of somatostatin analogue SMS 201-995 in treatment of human endocrine tumors. J Clin Invest 1994;93:1321-1325.

37 Volante M, Bozzalla-Cassione F, Papotti M. Somatostatin receptors and their interest in diagnostic pathology. Endocr Pathol 2004;15:275-291. 